\title{
LOS MAPAS CONCEPTUALES Y SU INFLUENCIA EN LA ENSENANZA PARA LA COMPRENSIÓN
}

\section{Conceptual Maps and Their Influence on Teaching for Understanding}

Fecha de recepción: 26 de noviembre de 2017

Fecha de aprobación: 12 de abril de 2018

Libia Andrea Rodríguez Garzón* Manuel Antonio Rueda Vargas**

Artículo de Reflexión

\section{Resumen}

Este trabajo presenta una experiencia educativa con estudiantes del grado sexto de la Institución Educativa Técnica Pablo VI del municipio de Sotaquirá y grado séptimo de la Institución Educativa Sergio Camargo de Miraflores en Boyacá, en el área de Ciencias Naturales y Educación Ambiental, sociales, sucedida durante el primer semestre del año 2017. Se contrastan los resultados del primer y segundo período en el área, una vez aplicada la estrategia propuesta.
El objetivo fundamental es la comprensión significativa del conocimiento científico a través del organizador gráfico mapa conceptual, aplicado en el diseño y desarrollo de unidades didácticas estructuradas para ciencias naturales de grado sexto, las cuales sustentan la metodología de Investigación-Acción implementada para la ejecución de la propuesta.

Se sustenta en los trabajos de Joseph Novak (1992), sobre mapas conceptuales; el aprendizaje significativo
* Institución educativa pablo VI de Sotaquirá - BoyacáColombia andrearodriguezgarzon@ hotmail.com

** Institución educativa. Sergio Camargo. MirafloresBoyacá- Colombia leunam328@gmail.com 
de David Ausubel (1963); y los aportes epistemológicos de David Perkins (2010) respecto a la Educación para la comprensión, y de Julián de Zubiría Samper (2006) en cuanto a modelos pedagógicos y el sentido de la educación.

Con la implementación de unidades didácticas estructuradas a partir de

\section{Abstract}

This work presents an educational experience with students of the sixth grade of the Pablo VI Educational Institution in Sotaquirá(Boyacá, Colombia), and seventh grade of the Sergio Camargo Educational Institution in Miraflores (Boyacá, Colombia) in the area of Natural Sciences and Environmental Education, during the first semester of the year 2017. The results of the first and second period in the area are contrasted once the proposed strategy has been applied. The fundamental objective is the significant understanding of scientific knowledge through conceptual maps, applied in the design and development of structured didactic units for sixth grade in natural sciences, which support the Research - Action methodology implemented mapas conceptuales, se promueve un conocimiento significativo de las ciencias naturales en la institución objeto de estudio, permitiendo a los estudiantes no sólo aprender sino también hacerlo comprensivamente.

for the proposal. It is based on the works of Joseph Novak (1992), on conceptual maps; Meaningful learning, by David Ausubel (1963); and the epistemological contributions of David Perkins (2010) regarding Education for Understanding, and Julián de Zubiría Samper (2006) regarding pedagogical models and the meaning of education. With the implementation of structured didactic units based on conceptual maps, a significant knowledge of the natural sciences is promoted in the institution under study, allowing students not only to learn, but also to do it comprehensively.

Keywords: Education, Strategy, Meaningful understanding, Conceptual map, Students, Research 


\section{Introducción}

Los métodos de enseñanza a lo largo de los años se han creado para apoyar el desarrollo de las mallas curriculares, a través de estrategias y metodologías que permitan una verdadera comprensión, retención y aplicación de los saberes que median la actividad escolar, más aún cuando los educandos -y la misma IEson evaluados por entidades Nacionales en los grados de $3^{\circ}, 5^{\circ}, 7^{\circ}, 9^{\circ}$ y $11^{\circ}$. Justo en la mitad de estos grados evaluados, se da una transición importante para desarrollar el tema objeto del presente proyecto. La naturaleza de las áreas y asignaturas se hace cada vez más compleja y los estudiantes requieren mejorar sus habilidades cognitivas. La brecha entre comprender y aplicar lo aprendido crece en la medida que se avanza hacia niveles superiores.

Antes de abordar la Enseñanza para la Comprensión, se considera pertinente recordar cómo se puede encontrar su vínculo con el aprendizaje significativo:

Si el aprendizaje es significativo, la estructura cognitiva está constantemente reorganizándose por diferenciación progresiva y reconciliación integrativa $y$, en consecuencia, los mapas trazados boy serán distintos de los trazados mañana. El profesor en vez de preocuparse por atribuir una puntuación al mapa trazado por el alumno, debe procurar interpretar la información dada por el mismo en el mapa con el fin de obtener evidencias de la existencia de aprendizaje significativo. (Moreira, 2013)

Lo anterior significa que se debe potenciar en el estudiante la capacidad para inferir, tanto como la de comunicar. El mapa reflejará su interpretación del conocimiento. Ese ha sido el trabajo con el grupo objetivo; poder elaborar mapas conceptuales que reflejen la comprensión de los temas vistos en ciencias naturales.

La enseñanza para la comprensión puede resumirse en el siguiente texto:

Comprender también es más que una habilidad rutinaria bien automatizada. El alumno que hábilmente resuelve problemas de física o escribe párrafos con oraciones tópicas puede no comprender casi nada de física, de escritura o de aquello acerca de lo que escribe. Aunque el conocimiento y la babilidad pueden traducirse como información y desempeño rutinario a mano, la comprensión se escapa de estas normas simples. Entonces ¿qué es la comprensión? [...] En pocas palabras, comprender es la babilidad de pensar y actuar con flexibilidad a partir de lo que uno sabe. [...] Por contraste, cuando un estudiante no puede ir más allá de la memorización y el pensamiento y la acción rutinarios, esto indica falta de comprensión. (Perkins, 1999 cp2)

Desde la experiencia docente se puede afirmar que lo expuesto tiene plena validez durante la interacción docenteestudiante en el aula de clase. Allí, para el grupo objetivo seleccionado (estudiantes de grado $6^{\circ}$ ), el éxito pedagógico-asociado equivocadamente con la valoración final- se reduce a cumplir los mínimos exigidos recurriendo a la memorización como
Si el aprendizaje es significativo, la estructura cognitiva está constantemente reorganizándose por diferenciación progresiva y reconciliación integrativa $y$, en consecuencia, los mapas trazados hoy serán distintos de los trazados mañana. 
estrategia para afrontar la validación de los conocimientos. ¿Pero realmente se ha aprendido?

Observando los contenidos curriculares del área de Ciencias Naturales y Educación Ambiental y Ciencias Sociales para los grados de referencia, se concluye que son muchos temas por abordar en el año lectivo, pero también que pueden mejorar ese «poco tiempo», la didáctica para ese abordaje de temas, y principalmente su comprensión, retención y aplicación.

En este punto, una vez se concluye que el objetivo fundamental para la IE desde su modelo pedagógico es lograr que el estudiante comprenda realmente lo que recibe en el aula y lo que debe repasar y producir en casa, se analiza qué tipo de herramienta puede incorporarse en las prácticas de aula para lograr que los alumnos puedan acceder de una manera diferente a los contenidos de las ciencias naturales y las ciencias sociales. Se escoge el mapa conceptual como el mejor instrumento para la representación de algunas temáticas, y como fundamento para la elaboración de unidades didácticas estructuradas para éstas áreas, las cuales se presentarán de manera extensa a las directivas institucionales para que se estudie también su incorporación en las otras áreas fundamentales y optativas.

\section{Reflexión}

Todos los días el quehacer docente está enfocado en lograr que se comprenda lo que se imparte. Más aún, en que esos conocimientos sirvan al estudiante para resolver situaciones de toda índole. En nuestro medio, la educación fundamenta su quehacer en alcanzar resultados superiores en las pruebas de Estado, a través de didácticas que faculten al estudiante para responder pruebas de manera eficiente.

Pero siempre está presente una pregunta: ¿en realidad los estudiantes comprenden lo que reciben en el aula de clase? ¿Comprenden qué tienen que hacer por su cuenta fuera del ella? ¿se comprende 'para cumplir' o 'para ser feliz’?

Y más preocupante aún: "Pareciera que se enseña y se estudia porque bay que estudiar y hay que enseñar. Lo más grave del caso es que -sin finalidades $e$ intenciones educativas claras $y$ previamente definidas- no es posible pensar ni actuar pedagógicamente." (De Zubiría Samper J. , Los modelos pedagógicos: Hacia una pedagogía dialogante, 2006 p31)

Los proyectos educativos institucionales se construyen para presentar a la comunidad educativa institucional y extensa (padres de familia, entorno social) las líneas gruesas o modelo pedagógico que se ha escogido como camino para resolver los análisis que arrojan las matrices DOFA en las cuales se condensan problemáticas y oportunidades para "formar integralmente a niños, niñas, jóvenes $y$ adultos de Sotaquirá y Miraflores desarrollando competencias en un ambiente de responsabilidad, respeto, liderazgo y calidad educativa, orientando su proyecto de vida que garantice un alto nivel de desempeño intelectual y laboral". IET Pablo VI e 


\section{IE Sergio Camargo.}

Sin embargo, una cosa es atender resultados institucionales, tener plantas docentes cualificadas por experiencia o por tiempo (que no es lo mismo), y otra poder reconocer y medir si los estudiantes comprenden de verdad los temas y contenidos que reciben.

En este sentido, todos los modelos que orbitan la educación en el país, desde la Escuela Tradicional, la Escuela Activa hasta los enfoques constructivistas, pasando por Escuela Nueva, afirman la necesidad de "concebir al niño como un ser independiente y no como un «adulto en miniatura»". (De Zubiría Samper J. , 2006 p107)

Por lo anterior, se deja la siguiente inquietud, con la cual se aborda el tema objeto del presente artículo, postulada por Alberto Merani y citada por investigadores contemporáneos:

"Somos seres histórica y culturalmente determinados. De este modo los individuos somos por nacimiento, nos mantenemos en el ser histórico por duración y realizamos nuestro ser en las circunstancias socioculturales en que nos toca vivir" (De Zubiría Samper J. , Los modelos pedagógicos: Hacia una pedagogía dialogante, 2006 p199).

Según los estándares para lengua castellana:

[...] "El lenguaje posee doble valor: subjetivo y social, por lo que apoya la formación de individuos autónomos, capaces de interpretar, comprender y transformar su contexto, a la vez que les permi- te reconocerse como seres únicos con características similares, pero con igualdad de derechos, responsabilidades y potencialidades; en consecuencia, la importancia de despertar el interés y fomentar en los estudiantes la apropiación de las habilidades orales y escritas, ya que estas son herramientas necesarias en la construcción de mundos posibles, para que cada estudiante se apropie de su papel en el mundo que lo circunda”. (Ministerio de Educación Nacional, 2006, p18)

Ese doble valor -subjetivo y socialque posee el lenguaje (Ministerio de Educación Nacional, 2006, p18) sugiere la importancia de entender el contexto institucional del estudiante, y más allá el familiar, donde se gesta la transformación del contexto personal y el desarrollo de habilidades desde la capacidad de entender el mundo a través de los textos.

Desde esta perspectiva, aunque la base de análisis del presente escrito se centra en las ciencias naturales y sociales, ambas tienen completa incidencia -y las demás áreas fundamentales y optativasdesde la lengua castellana, en la que se encuentra la base del modelo de Enseñanza para la Comprensión:

"[...] "Los procesos: comprensión, interpretación y producción, suponen la presencia de actividades cognitivas básicas como la abstracción, el análisis, la síntesis, la inferencia, la inducción, la deducción, la comparación, la asociación. Así entonces, una formación en lenguaje que supone el desarrollo de estos procesos mentales en interacción con el contexto socio-cultural,
"El lenguaje posee doble valor: subjetivo y social, por lo que apoya la formación de individuos autónomos, capaces de interpretar, comprender y transformar su contexto, a la vez que les permite reconocerse como seres únicos con características similares, pero con igualdad de derechos, responsabilidades y potencialidades 
El enfoque pedagógico de la Enseñanza para la

Comprensión pretende ser un marco de referencia que explica cómo construir comprensiones e interpretaciones profundas y la importancia que éstas tienen para el desarrollo del pensamiento no sólo posibilita a las personas la inserción en cualquier contexto social, sino que interviene de manera crucial en los procesos de categorización del mundo, de organización de los pensamientos $y$ acciones, $y$ de construcción de identidad individual y social". (Ministerio de Educación Nacional, 2006, p21)

Partiendo de la normatividad educativa nacional, para el sector rural en el departamento de Boyacá -más específicamente en los municipios de Sotaquirá y Miraflores- se pensó en un estudio que permitiese mejorar la situación problema, vista desde las condiciones individuales de éstas comunidades educativas. Para ello se optó por la didáctica Enseñanza para la Comprensión, teniendo en cuenta que ésta busca mejorar la conexión que debe existir entre la interpretación y la producción oral y escrita, y -por endepromover un desempeño eficiente de los estudiantes en los procesos en el aula que les permita entender su contexto y mejorar sus condiciones sociofamiliares con una perspectiva de avance académico posible y sostenible en el tiempo.

El enfoque pedagógico de la Enseñanza para la Comprensión pretende ser un marco de referencia que explica cómo construir comprensiones e interpretaciones profundas y la importancia que éstas tienen para el desarrollo del pensamiento, permitiéndole al estudiante resolver problemas y situaciones de manera flexible, coherente con lo aprendido y acorde con sus necesidades, diferencias e inquietudes; así mismo, en la producción oral y escrita se implementan actividades contextualizadas a sus intereses y se aprovechan los recursos y espacios que ofrece el medio, en procura de una mejor participación, desarrollo de potencialidades, expectativas sociales con respecto a la convivencia y su proyecto de vida $-\mathrm{y}$ en consecuenciaun proceso de formación humana con bases sólidas. (Perkins, Enseñanza para la Comprensión EpC, 2010)

Las particularidades de la comunidad educativa objeto de estudio pueden encontrar en la EpC la motivación que se ha perdido en el proceso de enseñanza-aprendizaje, porque permite superar que los estudiantes se queden con dudas frente a los contenidos recibidos que no entienden, por miedo a los juicios de compañeros o el docente. La construcción promovida por el modelo EpC es de corte colaborativo y cooperativo, y la dinámica que se incorpora con el uso de la herramienta mapa conceptual, genera un ambiente si no perfecto-ideal para aprender con alegría y efectividad.

\section{Preguntas cortas y problemas breves}

Pese a que la educación en el departamento de Boyacá está considerada como la mejor del país, dado que sus resultados están desde hace varios años por encima de la media nacional, se debe dar una mirada más profunda a lo que sucede dentro de las instituciones educativas, y más aún al diseño y aplicación de los currículos y las estrategias de aprendizaje en las diferentes áreas básicas $\mathrm{O}$ 
fundamentales. Cabe preguntar ¿por qué -si por resultados se está mejor que el resto del país- no resulta coherente el nivel de preparación de las personas (estudiantes) que logran su paso a la educación superior? También es visible en la cotidianidad docente encontrar falta de motivación por el estudio, desinterés por asumir responsabilidades y métodos de estudio ineficientes.

Lo anterior, por mencionar lo que sucede «dentro» del aula. Las circunstancias familiares también influyen de manera determinante en los avances y resultados de los estudiantes. Finalmente, son muchos los estudiantes que dejan de aprender, pero no por dificultades de aprendizaje o necesidades educativas específicas. Muchos estudiantes no aprenden porque no alcanzan a llegar a tiempo a la institución educativa, ya sea porque viviendo cerca no lo hacen, porque ingresan a la institución educativa pero no al aula de clase, porque ingresan al aula y deciden no aprender, o porque no alcanzan a llegar al colegio por tener que cubrir grandes distancias desde sus hogares.

\section{El lugar de la experiencia}

La Institución Educativa Técnica Pablo VI del municipio de Sotaquirá y Sergio Camargo de Miraflores en Boyacá en adelante IE-, en funcionamiento hace cuatro décadas, es una de esas instituciones conformada por muchas pequeñas sedes (14 contada la principal) ubicadas prácticamente todas en zona rural. En su totalidad, la comunidad educativa (más de 1100 estudiantes) se encuentra en los estratos 1 y 2 , con herencia en el trabajo agrícola y explotación ganadera. Se seleccionaron los grados sexto (IET Pablo VI de Sotaquirá) y séptimo (IE Sergio Camargo de Miraflores) como grupo objetivo para el desarrollo de la experiencia.

Las dos instituciones dentro de sus proyectos educativos institucionales tienen como propuesta pedagógica "[...] la Pedagogía activa y participativa, que integra y enfatiza otros modelos, principalmente el Constructivismo, buscando que la comunidad educativa direccione el proceso enseñanzaaprendizaje colaborativo bacia el cumplimiento de las políticas y metas en atención a la diversidad. A su vez, se menciona que " $A$ partir del año 2012, se tomó como ENFOQUE pedagógico el marco de ENSEÑNANA PARA LA COMPRENSIÓN como referencia del modelo del constructivismo". (Institución Educativa Técnica Pablo VI de Sotaquirá).

Como se mencionó anteriormente, aunque los resultados institucionales en pruebas de Estado infieren un buen nivel institucional en todas las áreas, se ha detectado desde la individualidad estudiantil que existen falencias para que los estudiantes puedan desarrollar todo su potencial, disfrutando del aprendizaje en el camino. El área escogida es Ciencias naturales.

\section{Contexto institucional objeto de estudio}

Institución Educativa Técnica Pablo Sexto (Sotaquirá)

La Institución Educativa Técnica Pablo Sexto ubicada en el municipio la Pedagogía activa y participativa, que integra y enfatiza otros modelos, principalmente el Constructivismo, buscando que la comunidad educativa direccione el proceso enseñanza-aprendizaje colaborativo hacia el cumplimiento de las políticas y metas en atención a la diversidad. 
de Sotaquirá en la zona centro de Boyacá. Presta servicio en los niveles de preescolar, básica primaria y secundaria, media académica y técnica -con especialidades en Comercio, Procesamiento y Ciencias Naturales-. Cuenta con 15 sedes, y reúne 1000 estudiantes atendidos por un equipo interdisciplinario conformado por una rectora, tres coordinadores, 23 docentes de secundaria, 25 docentes de primaria, 7 administrativos y una psicoorientadora escolar. Se ofrece programa de educación para jóvenes y adultos con el operador Transformemos.

La población sotaquireña se encuentra entre los estratos uno y dos, y la actividad económica gira en torno a labores relacionadas con agricultura y ganadería principalmente.

\section{Institución Educativa Sergio Camargo (Miraflores)}

La Institución Educativa Sergio Camargo se encuentra ubicada en el municipio de Miraflores (Boyacá). Presta sus servicios en los niveles de preescolar, básica (primaria y secundaria), media vocacional académica con oferta técnica en las especialidades de Comercio y Sistemas Tiene 8 sedes para una población aproximada de 1100 estudiantes, atendidos por un equipo de 48 docentes, 9 administrativos, 4 directivos docentes y la psicoorientadora escolar. Se ofrecen programas de educación para jóvenes y adultos como CEDEBOY y Telesecundaria.

\section{Metodología}

Se escogió el modelo de InvestigaciónAcción porque el centro de atención se encuentra en el mejoramiento de las prácticas y su transformación positiva, se quiere cambiar una situación experimentada como problémica, y posibilitar la comprobación de ideas en la práctica pedagógica para mejorarla, acrecentando los conocimientos sobre el currículum, la enseñanza y el aprendizaje. (Pereyra, 2008).

En una primera fase se hizo un diagnóstico institucional a partir de la caracterización del modelo pedagógico consignado en el Proyecto Educativo Institucional, y los enfoques que se plantean, frente a la realidad del diseño y aplicación de los contenidos temáticos. Se detectaron las falencias de los estudiantes para la comprensión de temáticas relacionadas con el área de ciencias naturales y ciencias sociales.

Como segunda fase se seleccionaron los temas que se trabajarían en las unidades planteadas con los mapas conceptuales, correspondientes al Primer Período de la vigencia 2017, de los Planes de Área de Ciencias Naturales Grado Sexto (IET Pablo VI) y Ciencias Sociales Grado Séptimo (IE Sergio Camargo).

En una tercera fase se hace toda la indagación sobre la construcción de los mapas conceptuales para explicarlo a los estudiantes del grupo objetivo desde el valor y necesidad de elaborar procesos de jerarquización de los contenidos recibidos. Se trabaja sobre los elementos del mapa como 'conceptos', 'palabras de enlace', 'proposiciones', 'líneas y flechas de enlace' y 'conexiones cruzadas', y se ofrecen ejemplos aclaratorios desde 
vivencias cotidianas.

En la fase cuatro se elaboran las unidades didácticas estructuradas y comienza su aplicación. Los contenidos se seleccionan de los planes de área de cada grado correspondientes al primer período lectivo.

Posteriormente se inicia la validación de resultados, fase que se encuentra parcialmente terminada por no haber concluido el segundo período académico de la presente vigencia. Sin embargo, la información contrastada arroja preliminarmente una mejora en la forma como se asimilan los temas, su comprensión y los resultados de las mediciones y valoraciones de progreso aplicadas. Se ha constatado incremento en la dinámica colaborativa en clase.

\section{Conclusiones}

El jerarquizar el conocimiento y priorizar los temas a trabajar, permitirán que el estudiante de grado sexto de la IE Pablo VI del municipio de Sotaquirá y Sergio Camargo de Miraflores tenga una lectura más activa y entienda mejor su razón de ser dentro del aula.

Es indispensable que los tópicos del área de ciencias naturales estén focalizados, de tal manera que el estudiante pueda tener una interacción directa de forma subjetiva y objetiva con el conocimiento.

Al ser la enseñanza para la comprensión una parte del constructivismo, posibilita al docente dinamizar dentro del aula la jerarquización del conocimiento por medio de los mapas conceptuales.

La jerarquización del conocimiento a través del mapa conceptual trae consigo la posibilidad de flexibilizar el conocimiento y brinda verdaderas herramientas de interacción entre el estudiante y el docente, toda vez que vincula los saberes previos del estudiante con los tópicos que establece el docente para el desarrollo de su clase.

La comunicación no es unidireccional, como siempre se ha establecido, sino que encaja en la practicidad y dinamismos del docente, convirtiendo el proceso bidireccional, en el cual el docente se enriquece de las apreciaciones del estudiante y este último comprende sus limitaciones.

\section{Referencias}

De Zubiría SAmper, J. (2006 p107). Los modelos pedagógicos: Hacia una pedagogía dialogante. Bogotá, D.C., Colombia: Cooperativa Editorial Magisterio Colección Aula Abierta.

De Zubiría SAmper, J. (2006 p199). Los modelos pedagógicos: Hacia una pedagogía dialogante. Bogotá, D.C.: Cooperativa Editorial Magisterio - Colección Aula Abierta. 
De Zubiría SAmper, J. (2006 p31). Los modelos pedagógicos: Hacia una pedagogía dialogante. Bogotá: Cooperativa Editorial Magisterio - Colección Aula Abierta.

Institución Educativa Técnica Pablo VI Sotaquirá. (2016 p18). Currículo, Propuesta Pedagógica. Proyecto Educativo Institucional 2016. Sotaquirá, Boyacá, Colombia: IE Pablo VI.

Institución Educativa Técnica Pablo VI Sotaquirá. (2016 p7). Proyecto Educativo Institucional. Misión. Sotaquirá, Boyacá, Colombia: IE PabloVI.

Ministerio de Educación Nacional. (2006, p18). Estándares Básicos de Competencias. Documento No.3. MEN.

Ministerio de Educación Nacional. (2006, p21). Desempeños de Competencias. Documento No.3. MEN Colombia.

Moreira, M. A. (25 y 26 de marzo de 2013). Mapas conceptuales y aprendizaje significativo. Obtenido de mapasesp.pdf: https://www.if.ufrgs.br/ moreira/ mapasesp.pdf

Pereyra, M. (14 de octubre de 2008). Redacción 1 - Facultad de Ciencia Politica y RR.II de la Universidad Nacional de Rosario. Obtenido de La Investigación Acción en Educación: http://www.fcpolit.unr.edu.ar/ redaccion1/2008/10/14/la-investigacion-accion-en-educacion/

Perkins, D. (1999 cp2). ¿Qué es la comprensión? Obtenido de La enseñanza para la comprensión. Fragmento de M. Stone Wiske (Compil.): http:// www.medfamco.fmed.edu.uy/Archivos/pregrado/Ciclo_Introductorio/ Materiales/que_es_la_comprensi_n_1.pdf

Perkins, D. (2010). Enseñanza para la Comprensión EpC. Obtenido de http:// www.slideshare.net/cavalos82/la-enseanza-para-la- comprensin 